\title{
Electromagnetic navigational bronchoscopy for diagnosing peripheral lung lesions in lung transplant recipients: a single- center experience
}

\author{
Tanmay S. Panchabhai, Sreeja Biswas Roy, Nikhil Madan, Hesham Abdelrazek, Vipul J. Patel, Rajat \\ Walia, Ross M. Bremner \\ Norton Thoracic Institute, St. Joseph's Hospital and Medical Center, Phoenix, AZ, USA \\ Correspondence to: Tanmay S. Panchabhai, MD, FACP, FCCP. Department of Pulmonary Medicine, Norton Thoracic Institute, St. Joseph's Hospital \\ and Medical Center, Phoenix, AZ, USA. Email: tanmay.panchabhai@dignityhealth.org.
}

Submitted Apr 03, 2018. Accepted for publication Jul 16, 2018.

doi: $10.21037 /$ jtd.2018.07.81

View this article at: http://dx.doi.org/10.21037/jtd.2018.07.81

\section{Introduction}

Lung nodules are common in lung transplant recipients (1), but the immunosuppressed status of these patients means that diagnosing these nodules is urgent. Differential diagnoses in lung transplant recipients with lung nodules include infection (i.e., bacterial, fungal, mycobacterial), malignancy [e.g., bronchogenic, post-transplant lymphoproliferative disorder (PTLD), or metastatic malignancy], and hemorrhage after previous transbronchial biopsies $(1,2)$. Infections from Pseudomonas and Aspergillus species are the most common causes of lung nodules in these patients (3). Such lesions can drastically affect management of lung transplant recipients, especially when resistant infection, PTLD, or bronchogenic carcinoma is suspected $(1,2,4)$. Making a timely diagnosis is of paramount importance.

Tissue sampling by computed tomography (CT)guided biopsy may not be justified due to the high risk of pneumothorax, which poses special complications in bilateral lung transplant recipients with "buffalo chest" phenomenon $(5,6)$, as well as the lower chances of isolating organisms on culture of CT-guided biopsies, especially in cases of consolidative lesions or with fungal infection $(7,8)$. The diagnostic yield of conventional bronchoscopy with bronchoalveolar lavage (BAL) and transbronchial lung biopsies in lung transplant recipients approximates $60 \%$ for infection (9). Electromagnetic navigational bronchoscopy (ENB), on the other hand, has been shown to have a high diagnostic yield (i.e., 74\%) in diagnosing suspected malignant lung lesions $(10,11)$, and complications such as pneumothorax are much less common (3\%). ENB for the diagnosis of peripheral lung lesions in lung transplant recipients has been reported in only one single-center report (4) and one case report (12). Because airway anatomy does not change after lung transplant, the procedural aspects of ENB remain the same in lung transplant recipients, although the etiology of peripheral lung lesions is significantly different as compared to general population. The aim of the present study was to evaluate the diagnostic yield and safety of ENB in lung transplant recipients with peripheral lung lesions.

\section{Methods}

After Institutional Review Board approval (PHXA-18-007471-18), we retrospectively reviewed our lung transplant database to collect data from all lung transplant recipients who underwent ENB for the diagnosis of peripheral lung lesions at Norton Thoracic Institute at St. Joseph's Hospital and Medical Center between January 01, 2016 and January 1, 2018. All cases of ENB are carried out under general anesthesia after endotracheal intubation. Requirement of patient informed consent was waived by the Institutional Review Board. We use the superDimension (Medtronic, Minneapolis, MN, USA) navigation system (version 7.1) for all ENB procedures, and radial probe endobronchial ultrasound (rp-EBUS) is used for confirmation. All procedures are done under fluoroscopic guidance. The ENB tools used included a 21-gauge needle, brush, triple-needle brush, and biopsy forceps. A local guided wash $(5 \mathrm{cc})$ with 
Table 1 Demographic characteristics of 10 lung transplant recipients who underwent electromagnetic navigational bronchoscopy

\begin{tabular}{|c|c|c|c|c|c|c|c|c|}
\hline Pt. No. & Age, sex & Type of LT & $\begin{array}{l}\text { Native lung } \\
\text { disease }\end{array}$ & $\begin{array}{l}\text { CMV } \\
(\mathrm{D} / \mathrm{R})\end{array}$ & $\begin{array}{l}\text { EBV } \\
(\mathrm{D} / \mathrm{R})\end{array}$ & $\begin{array}{l}\text { Time from LT } \\
\text { to ENB }\end{array}$ & Immunosuppression & $\begin{array}{l}\text { Symptoms and signs at } \\
\text { presentation }\end{array}$ \\
\hline 1 & 65 , male & BSLT & IPF & $+/-$ & $+/+$ & $\begin{array}{l}2 \text { years, } \\
7 \text { months }\end{array}$ & $\begin{array}{l}\text { Prednisone, FK, } \\
\text { everolimus }\end{array}$ & Malaise, fevers for 2 weeks \\
\hline 2 & 62 , male & BSLT & IPF & $+/-$ & $+/+$ & $\begin{array}{l}1 \text { year, } \\
7 \text { months }\end{array}$ & $\begin{array}{l}\text { Prednisone, FK, } \\
\text { MMF }\end{array}$ & Cough and worsening dyspnea \\
\hline 4 & 64 , male & BSLT & COPD & $+/+$ & $? /+$ & 10 months & $\begin{array}{l}\text { Prednisone, FK, } \\
\text { MMF }\end{array}$ & Cough and worsening dyspnea \\
\hline 5 & 75 , female & LSLT & IPF & $+/-$ & $? /-$ & $\begin{array}{l}5 \text { years, } \\
3 \text { months }\end{array}$ & $\begin{array}{l}\text { Prednisone, FK, } \\
\text { MMF }\end{array}$ & $\begin{array}{l}\text { Malaise, poor appetite, decline } \\
\text { in lung function }\end{array}$ \\
\hline 8 & 71 , male & BSLT & IPF & $-/-$ & $+/-$ & 2 years & $\begin{array}{l}\text { Prednisone, FK, } \\
\text { MMF }\end{array}$ & $\begin{array}{l}\text { Detected on CT done for } \\
\text { persistent decline in PFTs }\end{array}$ \\
\hline 9 & 73 , male & BSLT & COPD & $+/-$ & $+/+$ & $\begin{array}{l}1 \text { year, } \\
10 \text { months }\end{array}$ & $\begin{array}{l}\text { Prednisone, FK, } \\
\text { MMF }\end{array}$ & $\begin{array}{l}\text { Incidental lung nodule detected } \\
\text { on imaging }\end{array}$ \\
\hline 10 & 67 , male & RSLT & COPD & $+/+$ & $? /+$ & $\begin{array}{l}4 \text { years, } \\
5 \text { months }\end{array}$ & $\begin{array}{l}\text { Prednisone, FK, } \\
\text { MMF }\end{array}$ & $\begin{array}{l}\text { Cough, dyspnea on exertion, } \\
\text { decline in PFTs }\end{array}$ \\
\hline
\end{tabular}

?, status unknown; LT, lung transplant; BSLT, bilateral sequential lung transplant; LSLT, left single lung transplant; RSLT, right single lung transplant; IPF, idiopathic pulmonary fibrosis; BOS, bronchiolitis obliterans syndrome; COPD, chronic obstructive pulmonary disease; PPFE, pleuroparenchymal fibroelastosis; CMV, cytomegalovirus; D, donor; R, recipient; EBV, Epstein-Barr virus; ENB, electromagnetic navigational bronchoscopy; FK, tacrolimus; MMF, mycophenolate mofetil; CT, computed tomography; LLL, left lower lobe; SCC, squamous cell carcinoma; s/p, status post; PFTs, pulmonary function tests.

the extended working channel was obtained in all patients followed by a BAL; each was sent separately for culture and analysis. Specimens from the needle aspirates and brushings were not sent for culture to ensure availability of adequate sample for cytopathology. Linear EBUS is used to sample hilar and mediastinal lymph nodes where necessary. Rapid onsite evaluation (ROSE) is available in most cases. When imaging characteristics suggested infection, transbronchial biopsies were sent for culture as well.

We recorded the following data: lesion sampled, procedural tools used, ROSE results, and results from cytopathology and cultures. Demographic details of these patients, including lung transplant type, cytomegalovirus (CMV) status, Epstein-Barr virus (EBV) status, time between lung transplant and ENB procedure, immunosuppressive medications, and symptoms at presentation were also reviewed. We used descriptive statistics due to the small sample size and means were calculated for continuous variables.

\section{Results}

Between January 01, 2016 and January 01, 2018, 10 lung transplant recipients underwent ENB for peripheral lung lesions. Table 1 summarizes the demographic characteristics of these patients. Eight of 10 patients underwent bilateral sequential lung transplant; the other 2 patients underwent single lung transplant. This cohort included 7 men and 3 women, and the mean age was 64.3 years. The appearance of peripheral lung lesions ranged from 9 months after lung transplant to 5.25 years after lung transplant. In 2 patients (patient 5 and patient 10, Table 1), nodules developed in the native lung. The presenting symptoms that prompted CT imaging are listed in Table 1; most patients underwent CT 

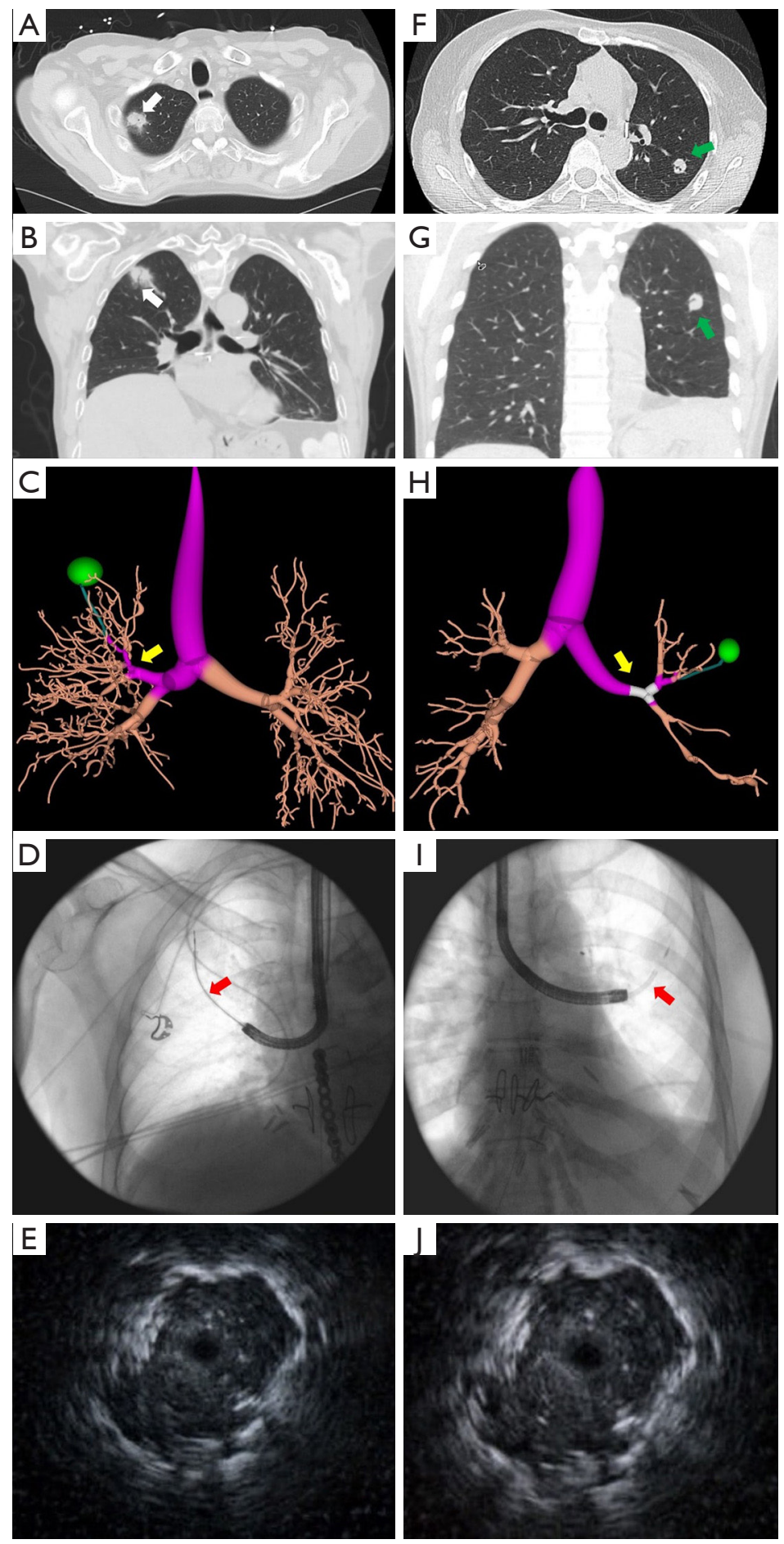

Figure 1 Computed tomogram of patient 2 shows the right upper lobe lesion in transverse (A) and coronal (B) views (white arrow); (C) shows the plan created for ENB with the superDimension navigation system (Medtronic, Minneapolis, MN, USA; yellow arrow). The fluoroscopic image with the extended working channel (D, red arrow) is also shown as well as the radial endobronchial ultrasound image (E) of the same lesion; (F,G) show the transverse and coronal CT images showing a left upper lobe nodule (green arrow) in patient 6; (H) shows the plan created for ENB (yellow arrow); (I) shows the fluoroscopic image with the extended working channel in place (red arrow); (J) depicts the radial endobronchial ultrasound image of the left upper lobe nodule. ENB, electromagnetic navigational bronchoscopy; CT, computed tomography. 
for cough and worsening dyspnea, followed by decline in pulmonary function tests. All 10 patients were on standard post-transplant immunosuppression, including tacrolimus, mycophenolate mofetil, everolimus, and prednisone. All patients were followed closely in post-lung transplant clinic in accordance with program protocols and had follow-up CT imaging at 3 months. The follow-up period for patients included in the study ranged from 24 months (patient 1) to 3 months (patient 10) based on the date and time when ENB was performed.

All ENB procedures were done under general anesthesia with endotracheal intubation, and rp-EBUS was used for lesion confirmation in all 10 cases. ROSE was available for use in 8 of 10 cases. All patients had a bronchus sign on pre-procedural CT, per ENB protocol (1.25-mm sections). Details of ENB in these 10 patients, including lesion size, tools used, preliminary diagnosis on ROSE, final diagnosis (on cytopathology or culture), and complications are listed in Table 2. The CT images, navigational bronchoscopy plans, fluoroscopic images, and radial EBUS images have been depicted in Figure 1. The mean size (based on largest dimension) of the nodules sampled on CT imaging was $20.5 \mathrm{~mm}$. Lung nodules presented either with solid mass lesions or mass-like consolidation. Seven of the 10 patients were diagnosed with infections-with the organisms being Aspergillus species ( $\mathrm{n}=3)$, Nocardia species $(\mathrm{n}=2)$, Mycobacterium avium-intracellulare $(\mathrm{n}=1)$, and Pseudomonas aeruginosa $(\mathrm{n}=1)$. In these 7 patients diagnosed with infections, cultures of the guided wash were diagnostic in all 7 patients, whereas the BAL was diagnostic in just 3 patients (Table 2). Culture of ENB-guided transbronchial biopsies were diagnostic only in 1 of 7 patients diagnosed with infections (patient 3). Two patients were diagnosed with squamous cell cancer (SCC); in 1 patient, the SCC was discovered in the native lung; in the other, SCC was found in the transplanted lung. Patient 7 was diagnosed with organizing parenchymal hemorrhage related to prior transbronchial lung biopsy (Table 2). ROSE was not diagnostic of the final pathology in 5 of 6 patients with lung nodules from infection. However, ROSE was diagnostic in both patients with SCC. All 7 patients with infections were treated aggressively with sensitivity-based antibiotics, and lung lesions resolved on follow-up imaging in all 7 patients. The number of patients included in this preliminary report was small, and hence tool-specific yields and factors affecting the same could not be assessed.

Patient 5 was noted to have an enlarging right upper lobe mass, with mediastinal adenopathy of the native right lung with idiopathic pulmonary fibrosis. She had previously undergone a right lower lobectomy for cavitary pulmonary aspergillosis, without evidence of malignancy on the lobectomy specimen. She underwent ENB with linear EBUS for mediastinal adenopathy. The ROSE for the mediastinal lymph nodes was non-diagnostic for malignancy; therefore, ENB was performed for the RUL nodule and ROSE was positive for malignant cells. On final pathology, samples from the $\mathrm{N} 2$ lymph node and from the RUL nodule were positive for SCC, and the patient went on to receive chemoradiation for grade IIIA SCC originating in her native lung with idiopathic pulmonary fibrosis. Patient 6 had previously undergone left lower lobectomy for Stage I SCC, but then developed a new left upper lobe nodule that was sampled via ENB. ROSE was positive for malignant cells, and final pathology revealed metastatic SCC. Staging with positron emission tomography and magnetic resonance imaging did not reveal metastatic disease elsewhere, and she went on to receive stereotactic body radiation therapy for the solitary LUL oligometastatic focus. Patient 7 was diagnosed with a lung nodule from prior hemorrhage related to transbronchial biopsy, as resolving focus of parenchymal hemorrhage was reported on pathology. When her images and history were reviewed, this site was found to be the location of a prior transbronchial lung biopsy (a finding described by others) (2). Her nodule had resolved on serial follow-up imaging. No patient experienced complications such as pneumothorax, bleeding, or need for prolonged mechanical ventilation, and all patients were discharged home on the same day as the ENB procedure.

\section{Discussion}

Peripheral lung lesions are particularly challenging to manage in lung transplant recipients. Diagnoses such as bronchogenic carcinoma, PTLD, and atypical infections from Nocardia or Mycobacteria require immediate attention due to the immunosuppressed status of these patients and the greater chance of these infections to spread. Current evidence supports the use of ENB for diagnosis of suspected malignant peripheral lung nodules in non-transplant recipients $(10,11)$. However, the use of ENB in lung transplant recipients has been reported in just one other study (4), in which the authors reported a diagnostic yield of $75 \%$ (9/12 cases) for all advanced diagnostic bronchoscopic techniques (i.e., ENB, linear EBUS, and rp-EBUS) diagnosing 6 pneumonia and 3 malignancies (4). Another case report describes the use of ENB to diagnose PTLD in 


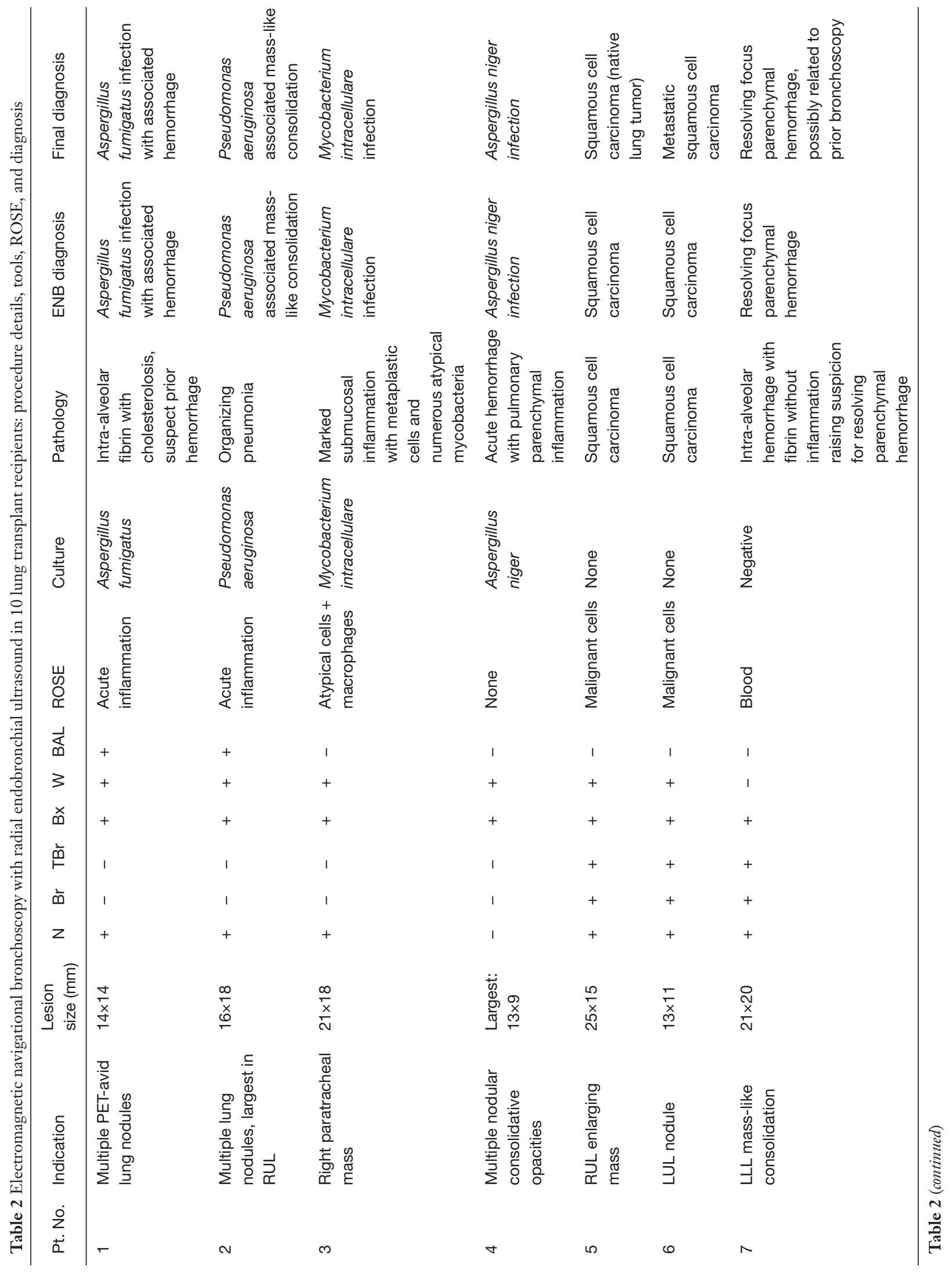




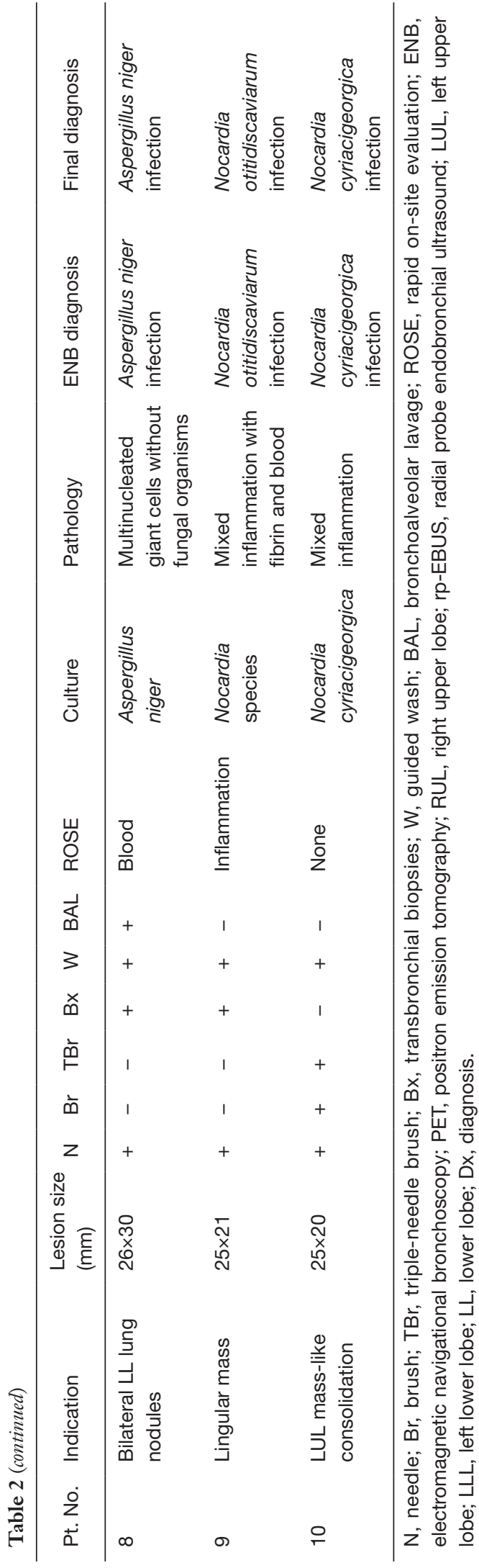

a lung transplant recipient (12).

In our cohort, ENB was useful in making a definitive diagnosis in 9 of 10 patients (diagnostic yield: $90 \%$ ). For the patient diagnosed with resolving parenchymal hemorrhage, a review of imaging and the timeline of previous procedures was more helpful in making the diagnosis; therefore, the ENB diagnosis was considered non-diagnostic. Similar to the findings reported by Cumbo-Nacheli and colleagues (4), we diagnosed 7 focal infections (70\%) and 2 malignant lesions (20\%). Moreover, when the final diagnosis was infection, the combined yield from the cultures of guided wash, BAL fluid, and biopsies was $100 \%$. The guided wash culture was positive in all 7 patients with infections, followed by the BAL that was positive in 3 of 7 patients, followed by culture of the transbronchial biopsies that was positive in 1 patient. Moreover, atypical organisms such as Nocardia species and atypical Mycobacteria were isolated on the guided wash but not on the BAL (Table 2). In these lesions, which were ultimately diagnosed as focal infections, pathology of needle aspirates/brushings and biopsies led to the diagnosis in just 1 of 7 patients.

Our preliminary observations suggest that ENB is a safe diagnostic modality for the diagnostic workup of peripheral lung lesions in lung transplant recipients, with a high diagnostic yield of both infection and malignancy. It can also be inferred from our results that the utility of ROSE in peripheral lung lesions with infection-like features is very limited. Our study has the following strengths: it is a singlecenter experience from a large lung transplant program, and we report the utility of diagnostic tools, ROSE, and culture in the lung transplant population. The limitations of this preliminary report include its retrospective nature, its small sample size, and a selection bias, as ENB was performed only on patients with a bronchus sign on pre-procedural CT. Further larger studies would better validate the diagnostic yield and complications of ENB in diagnosing peripheral lung lesions in lung transplant recipients.

\section{Acknowledgements}

None.

\section{Footnote}

Conflicts of Interest: The authors have no conflicts of interest to declare. 


\section{References}

1. Lee P, Minai OA, Mehta AC, et al. Pulmonary nodules in lung transplant recipients: etiology and outcome. Chest 2004;125:165-72.

2. Mehta AC, Wang J, Abuqayyas S, et al. New NoduleNewer Etiology. World J Transplant 2016;6:215-9.

3. Copp DH, Godwin JD, Kirby KA, et al. Clinical and radiologic factors associated with pulmonary nodule etiology in organ transplant recipients. Am J Transplant 2006;6:2759-64.

4. Cumbo-Nacheli G, Budev M, Machuzak M, et al. Usefulness of Advanced Diagnostic Bronchoscopic Techniques Among Lung Transplant Recipients With Pulmonary Nodules. Chest 2012;142:1092A.

5. Lee YC, McGrath GB, Chin WS, et al. Contralateral tension pneumothorax following unilateral chest tube drainage of bilateral pneumothoraces in a heart-lung transplant patient. Chest 1999;116:1131-3.

6. Sawalha L, Gibbons WJ. Iatrogenic "buffalo chest" bilateral pneumothoraces following unilateral transbronchial lung biopsies in a bilateral lung transplant recipient. Respir Med Case Rep 2015;15:57-8.

Cite this article as: Panchabhai TS, Biswas Roy S, Madan N, Abdelrazek H, Patel VJ, Walia R, Bremner RM. Electromagnetic navigational bronchoscopy for diagnosing peripheral lung lesions in lung transplant recipients: a singlecenter experience. J Thorac Dis 2018;10(8):5108-5114. doi: 10.21037/jtd.2018.07.81
7. de Bazelaire C, Coffin A, Cohen-Zarade S, et al. CTguided biopsies in lung infections in patients with haematological malignancies. Diagn Interv Imaging 2013;94:202-15.

8. Shi JM, Cai Z, Huang H, et al. Role of CT-guided percutaneous lung biopsy in diagnosis of pulmonary fungal infection in patients with hematologic diseases. Int $\mathrm{J}$ Hematol 2009;89:624-7.

9. Chan CC, Abi-Saleh WJ, Arroliga AC, et al. Diagnostic yield and therapeutic impact of flexible bronchoscopy in lung transplant recipients. J Heart Lung Transplant 1996;15:196-205.

10. Gildea TR, Mazzone PJ, Karnak D, et al. Electromagnetic navigation diagnostic bronchoscopy: a prospective study. Am J Respir Crit Care Med 2006;174:982-9.

11. Silvestri GA, Feller-Kopman D, Chen A, et al. Latest advances in advanced diagnostic and therapeutic pulmonary procedures. Chest 2012;142:1636-44.

12. Joshi D, Gildea T, Budev M. Diagnosis of Posttransplant Lymphoproliferative Disorder (PTLD) in a Lung Transplant Recipient Using Electromagnetic Navigational Bronchoscopy. Chest 2013;144:18A. 